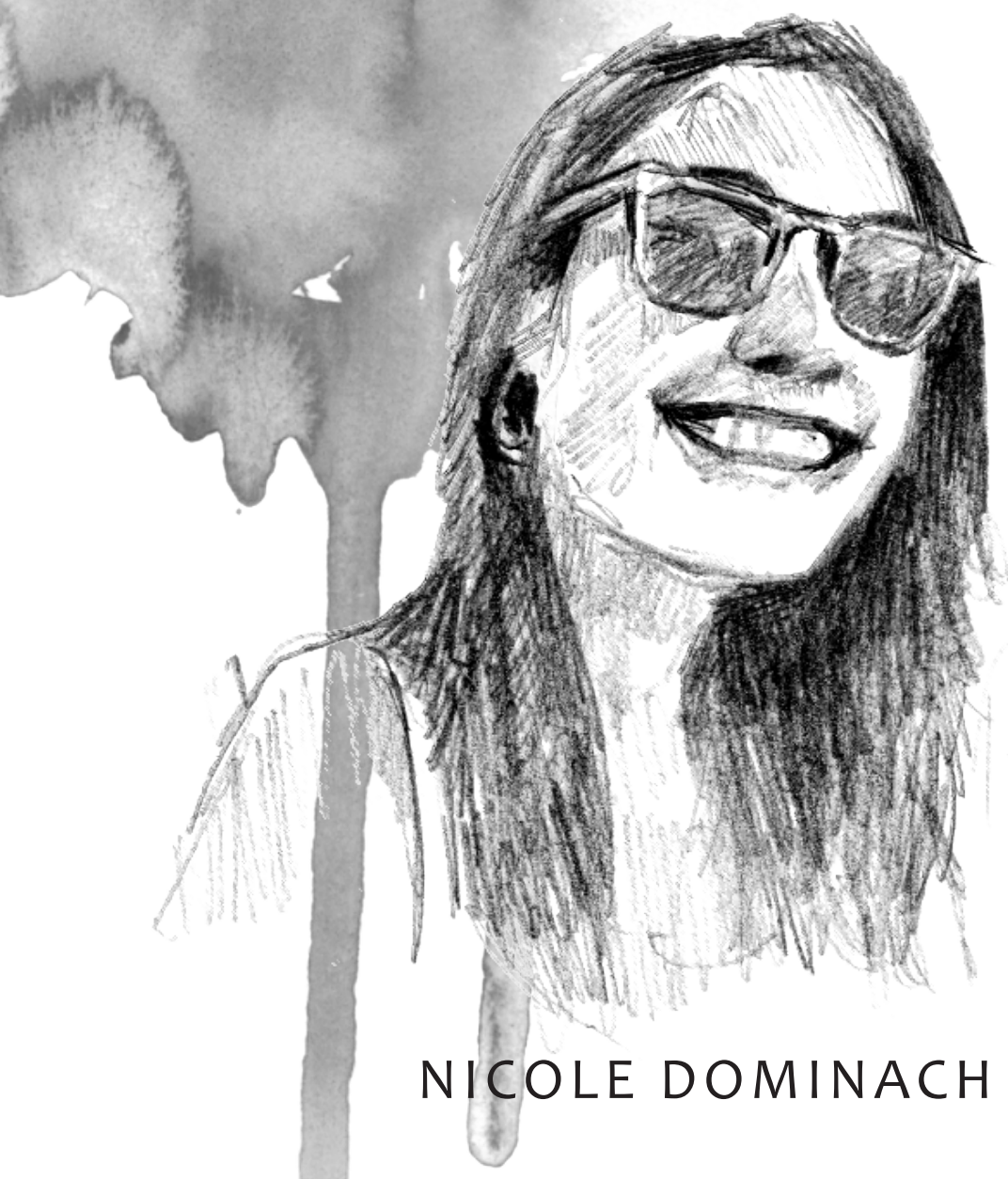

Nicole is a senior physical anthropology major with a chemistry minor. She is originally from Rochester, New York. Her favorite writer is Loren Eiseley, with "The Immense Journey" being her favorite of his works. After graduating from Pitt she plans on attending graduate school for Forensic Anthropology. 
FORBES \& FIFTH

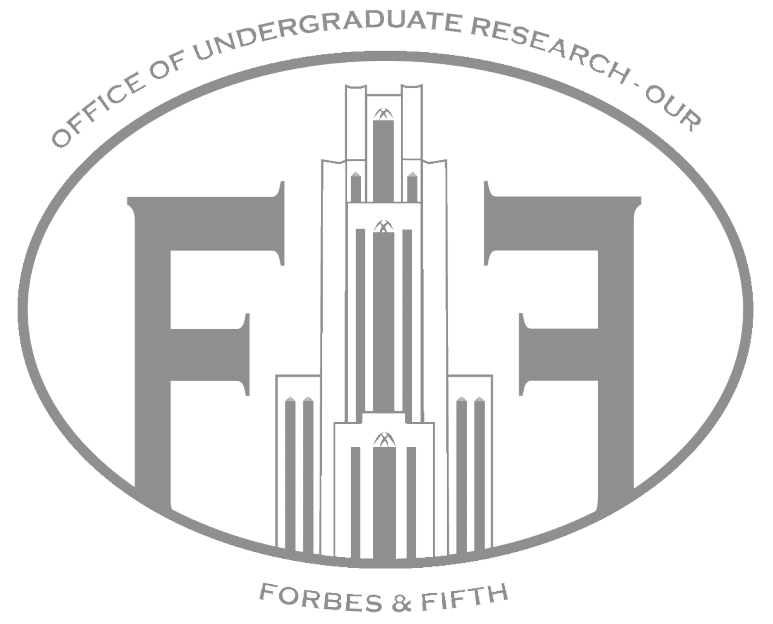




\section{An Overview of Sex Determination From the Adult Shoulder Girdle}

Sex determination is a key factor in the identification of individuals in both archaeological and forensic cases. Documentation of the use of the shoulder girdle to estimate sex is limited with the first study using all components of the shoulder girdle occurring in 1963 by Robert van Dongen. The pelvis and skull are the predominant osteological elements used in sexing a decedent. But, it is not always the case that these bones are found, thus other osteological elements must be used to determine sex. The bones that comprise the shoulder girdle - the scapula, clavicle, and humerus - can be examined in order to determine the sex of a decedent when neither the skull nor the innominate are present.

The determination of a decedent's biological sex is made via observing the differences in bone architecture and sexual dimorphism. Biological sex is important in the determination of other physical attributes of the deceased, such as race, stature, and age. This is due to the fact that physical attributes are population specific; therefore, a more masculine feature in one culture could be considered a feminine feature in another. The fact that sexing, along with all other physical attributes, is population specific has led to the realization that a "universal standard" for sexing cannot be developed. Thus the standards for estimation of sex as developed by Trotter using the Terry Collection and Dwight using Harvard Medical cadavers, are no longer applicable to the current population of the United States, current forensic cases or the populations of other countries.

The importance of post-cranial bones, other than the innominate, to estimate biological sex of a decedent can be overlooked at times. Yet, discovery of a completely intact skeleton is very rare. Thus, the use of post-cranial bones is beneficial to sexing when the skull and innominate are not present. There are multiple studies that even suggest that the skull is not superior to post-cranial elements when sexing an individual. Bernzbeitia in 1989 composed a study indicating that the radial head was better at sexing than the skull, and Robling and Ulbelaker in 1997 composed a study that developed results indicating that metatarsals may be superior at sexing to the skull. 


\section{Development of Shoulder Girdle Components}

The shoulder girdle is comprised of three bones: the clavicle, the scapula, and the humerus - primarily the proximal end. Each of these components develops and ossifies in different manners. The clavicle develops from two primary ossification centers and one secondary ossification center. The primary centers fuse around the time of birth to form the shaft of the clavicle. The secondary center is located at the medial clavicular epiphysis, from which almost all clavicular growth takes place. This secondary center of ossification is the last portion of bone to fuse in the human body, usually during one's mid-twenties as according to Burns, 2007 and can be seen in Figure I. As an individual ages, the sternal articular surface of the clavicle becomes porous and pitted as compared to the dense and undulated nature of the articular surface in youth.

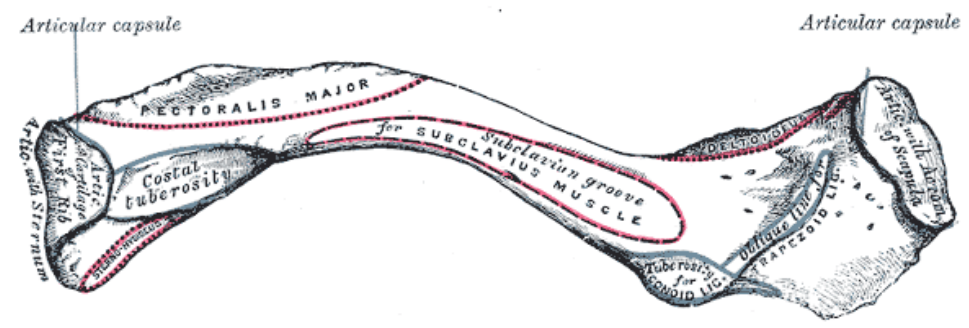

Figure I: Ossification Centers of Clavicle. Illustrates the three - two primary and one secondary - ossification centers of the clavicle. Image taken from http://upload.wikimedia. org/wikipedia/commons/f/fo/Gray201.png and originally taken from Gray's Anatomy of the Human Body, first published in 1858 .

The scapula develops from three parts via ossification centers of endochondral and intramembranous cartilage, as can be seen in Figure II. The primary center of ossification is located in the upper portion of the body of the scapula with endochondral ossification occurring laterally generating the glenoid fossa and medially to generate the vertebral border. Intramembranous ossification generates the majority of the scapular blade. A 
separate center of ossification forms the coracoid process during the first postnatal year with the fusion of the coracoid process to the body of the scapula between 15 and 17 years, according to Burns, 2007. The scapula develops from multiple secondary ossification centers along its vertebral border, inferior angle, acromion process, and glenoid fossa. These initially appear as tiny flakes. The scapula is completely ossified by one's early twenties according to Burns, 2007. As one ages, the glenoid fossa becomes more beveled and lipping occurs, as well as a sharp rim in some cases. These age related changes are more prominent on the skeleton's dominant side.

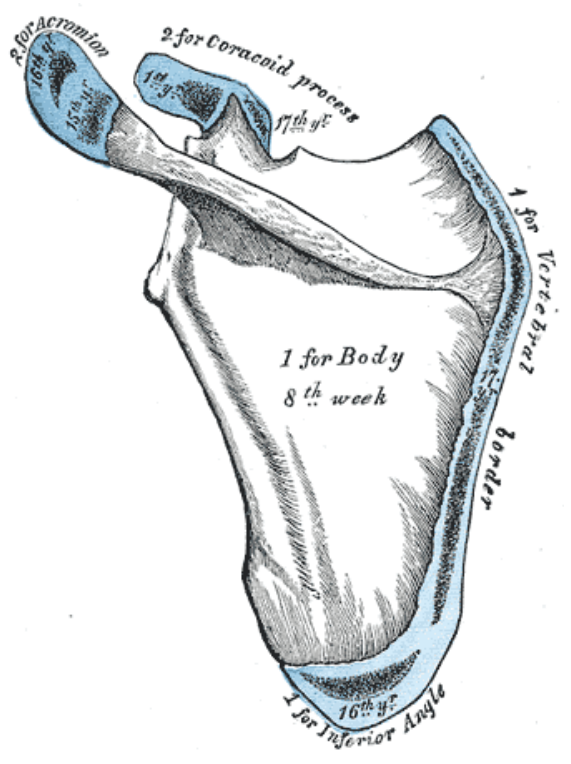

Figure II: Ossification Centers of Scapula. Illustrates the three main ossification centers of the scapula. The primary center is along the upper portion of the scapular body. There are multiple secondary centers of ossification. Image taken from http://upload.wikimedia. org/wikipedia/commons/7/79/ Gray206.png and originally taken from Gray's Anatomy of the Human Body, first published in 1858 .

The humerus originates via eight ossification centers - the head, shaft, lesser tubercle, greater tubercle, capitulum, trochlea, medial epicondyle, and lateral epicondyle - with the major centers forming the proximal and distal epiphyses (Burns, 2007, p. 96). This can be seen in Figure III. Fusion of the epiphyses varies by sex with females fusing earlier than males. The distal epiphyses fuse from 11 to 15 years in females and 12 to 17 years in males; the medial epicondyle fuses from 13 to 15 years in females and 12 to 17 years in males; and the proximal 
epiphysis fuses from 13 to 17 years in females and 16 to 20 years in males. Thus, by one's late teens to early twenties, the humerus should be fully fused. Age related changes to the humerus mainly take place at the humeral head in the form of osteoarthritis, resulting in bony projections and striations along the articular surface of the bone.

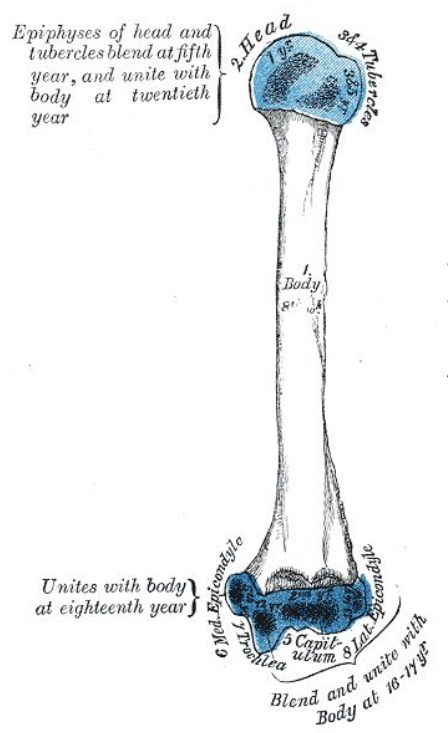

Figure III: Ossification Center of the Humerus. Illustrates the two main primary ossification centers of the humerus at the proximal and distal ends. Ossification centers also exist through the entire length of the humeral shaft. Image taken from http://upload.wikimedia.org/wikipedia/commons/o/06/Gray210.png and originally taken from Gray's Anatomy of the Human Body, first published in 1858.

\section{History of Sex Estimation for the Shoulder Girdle}

Use of the shoulder girdle to estimate sex of adult individuals is very limited in the history of forensic and physical anthropology, with the first study of the complete shoulder girdle occurring in the early 1960s. It was Thomas Dwight, the father of forensic anthropology in the United States, who was the first individual to develop methods for sex estimation using the components of the shoulder girdle. In his 1894 Shattuck Lecture, he stated that there are differences in the articular surfaces of skeletons which indicate sex. Dwight talked in depth about that scapula stating, "The shoulder-blade is an extremely variable bone. To me it is very interesting. I imagine that I shall surprise my hearers in speaking of its sexual characteristics as very remarkable" (Dwight, 1894, p.74). He then went on to explain two measurements of the scapula. The first was the scapular length measured on 198 specimens: 84 males and 39 
females. He found that any length less than $140 \mathrm{~mm}$ was most likely female and any length greater than $170 \mathrm{~mm}$ was most likely male. The second was the length of the glenoid fossa measured on 90 scapulae 63 males and 27 females - and found that that average male's length was $39.2 \mathrm{~mm}$ and the average female's length was $33.6 \mathrm{~mm}$. Out of the two measurements, Dwight found that the length of the scapula provided the most accurate procedure for sexing. The issues with Dwight's study are evident when one tries to reproduce it, for he does not indicate how measurements were taken or on whom, in regards to collection, they were taken. It is possible that the specimens were Harvard Medical cadavers and landmarks for measurements can be assumed, but accurate reproduction of his study is improbable. At the Shattuck Lecture, Dwight briefly mentioned the humerus and the clavicle. On the topic of the humerus, he stated that there are visual differences in sex, but gave no measurements or data. With the clavicle he stated that those of a female have a greater curve as compared with the straighter clavicle of a male and that the size of the articular surface may be of importance, but went no further on either subject.

In 1905, Dwight elaborated on his statement about the humerus from his 1894 lecture. He measured the maximum diameter of the humeral head - both its vertical and transverse measurements - of 200 fresh, white male and female specimens from the Harvard Medical School. Results were recorded only on the vertical measurements, and he found that the average male humeral head diameter was $48.76 \mathrm{~mm}$, the average female humeral head diameter was $42.67 \mathrm{~mm}$, and that there was an overlap between 45 and $46 \mathrm{~mm}$. Once again, Dwight did not mention the landmarks used for his measurements, yet they can be inferred. Still, this leads to inadequate results for comparison, if the study were to be tested. It is also important to note that these "standards" are biased toward the white population.

In 1932 and 1942, Aleš Hrdlička developed the next major studies of shoulder girdle components. His 1932 study, entitled The Principal Dimensions, Absolute and Relative, of the Humerus in the White Race, looked at different aspects of 4432 humeri of immigrants to the United States from the 1890s. This study included Caucasians from various nationalities, American Indians, and individuals of African descent. Both sexes and sides were used in this study. The dimensions Hrdlička 
looked at were length, length vs. height, mid-shaft diameter, mid-shaft strength, mid-shaft index (minimum mid-shaft diameter/maximum midshaft diameter), radio-humeral index (length of radius/length of humerus), and humero-femoral index (length of humerus/length of femur). The study found that the length of the humerus and mid-shaft circumference were greatest in Caucasians and lowest in individuals of African descent for females and greatest in Caucasians and lowest in American Indians for males. The mid-shaft strength and mid-shaft index was greatest in individuals of African descent and lowest in American Indians. The radio-humeral index was similar in American Indians and individuals of African descent in that it was greater than that of Caucasians. The humero-femoral index was similar in Caucasians and American Indians in that it was greater than that of individuals of African descent. In all dimensions males where larger than females, and in all groups the humero-femoral index is lower on the left side when compared to the right, probably due to right hand dominance in the human population. Hrdlička's study of humeral dimensions is beneficial in that it shows the difference in the humerus between sex, race, and nationality, but it does not give a set of standards for one to determine sex from. His paper is comparative study of the humerus, which is beneficial for educational purposes, but beyond that, it does not help in identifying the sex of an individual unless multiple specimens of different race, nationality, and sex are present.

Hrdlička's 1942 study looks at the scapula in a qualitative manner using morphological variations of the scapula body, axillary shape of the inferior angle, shape of the superior border, and shape of the scapular notch. His specimens were taken from the U.S. National Museum and are from Caucasians, American Indians, Ancient Egyptians, individuals of African descent, and Australian Aborigines. He also looks at two small juvenile samples using these qualitative methods. The study looked at differences between sex, side, race, and development that are predominantly of morphological value and causes of ontogenesis. He found that different populations have different scapular morphology and that female morphology is different from that of males. He also found that nationality allows for difference in scapular morphology, but fails to address differences between sides. Hrdlička states that:

"While data of this nature can never have the claim to the ac- 
curacy of measurements, and can only exceptionally be legitimately directly compared with those of other observers, their relative proportions are of value and throw light on many conditions which measurements alone will not be able to elucidate." (Hrdlička, 1942, p. 94)

Thus, he understands that quantitative methods are more accurate, but qualitative morphological studies can shed light on factors that may not be apparent during quantitative analysis. The issues with this method are that it is time consuming, must be done using one observer in order to obtain the most accurate data, and the multiple types for each scapular feature could become confusing

In 1963, Robert van Dongen became the first to address all components of the shoulder girdle in his study entitled The Shoulder Girdle and Humerus of the Australian Aborigine. He took a quantitative approach in measuring adult Aboriginal skeletons from the South Australian Museum. All specimens measured possessed at least one half of the innominate in order to establish the sex and no pathological or traumatic lesions. Two hundred and sixteen humeri (116 male and 100 female), 134 scapulae (69 male and 65 female), and 103 clavicles (50 male and 53 female) were measured in the study. Fifteen humeral measurements were taken with maximum length, diaphyseal index (minimum/maximum mid-shaft diameter), and torsion angle used for comparative measures. Nine quantitative scapular measurements were taken and six qualitative scapular measurements were taken with the quantitative measurements of maximum length, infraspinous length, breadth, and scapular index (breadth of scapula/max length) for comparative measures. Four clavicular measurements were taken, all quantitative. The results van Dongen obtained showed that the measurement of the humeral head was the most accurate at determining biological sex, with $80 \%$ accuracy. The maximum scapular length was most accurate at determining sex, but he failed to give an exact accuracy rate. However, he noted that the morphological features of the scapula are of little use in determination of sex. The minimum mid-shaft circumference was the most accurate dimension of the clavicle for sexing, but again he failed to give an exact accuracy. In comparison with other groups, van Dongen found that Australian Aborigines were within the normal range of human variation. His study is beneficial in that it was the first major study 
to focus on the shoulder girdle as a whole with very precise measurements that measure every aspect of each of the bones and that it was a population-specific study. The issues with van Dongen's study lie in its ability to replicate and use his thoroughness of analysis when working on an archaeological or forensic case when time is limited. It is also a comparative study of Aborigines to other populations, so it does not give a definitive method for determining sex in other populations.

In 1979, T. D. Stewart wrote Essentials of Forensic Anthropology: Especially as Developed in the United States, where he looked at various aspects needed for the identification of an individual. He addressed all components of the shoulder girdle, with the main focus on Dwight's scapulae and humeri data. Stewart focused on two features of the scapula: the scapular length and the length of the glenoid cavity. Using the data Dwight gave in his 1894 Shattuck Lecture, Stewart measured the scapular length from superior to inferior angle, although not specified by Dwight, on 90 right scapulae from the Terry Collection. Both African and Caucasian, male and female specimens were used. His results were consistent with Dwight's in that any length less than $14 \mathrm{~cm}$ were female, any lengths greater than $17 \mathrm{~cm}$ were male, and any lengths from $14-16 \mathrm{~cm}$ were indeterminate. Once again using the data from Dwight's 1894 Shattuck Lecture, Stewart measured the length of the glenoid cavity from the articular margin to the elevated bone of the supraglenoid tubercle, although again

"The benefits of these methods are that they have been repeated with consistent results, they are easy and fast to use, multiple ancestries were used, and they only require one bone to determine sex." not specified by Dwight, making sure no arthritic lipping was included in the measurement. $\mathrm{He}$ measured 50 right scapulas from the Terry Collection of both African/ Caucasian, and male/female specimens. Again Stewart's results were consistent with Dwight's. Glenoid cavity lengths measuring less than $34 \mathrm{~mm}$ were female, greater than $36 \mathrm{~mm}$ male, and between 34 and $36 \mathrm{~mm}$ indeterminate. The benefits of these methods are that they have been repeated with consistent results, they are easy and fast to use, multiple ancestries were used, and they only require one bone to determine sex. The issues with Stewart's 
methods are that the standard was based on individuals from the late $19^{\text {th }}$ and early $20^{\text {th }}$ century industrial working class, and they may not be applicable to foreign populations, pre-historic populations, or similar populations in modern times.

In testing the humerus, Stewart used Dwight's results from his 1905 study on the vertical humeral head diameter. He used 100 right humeri from the Terry collection consisting of both African and Caucasian, and male and female dry specimens. Stewart obtained results similar to that of Dwight's, but not as close as with the scapular features. He found a larger range of uncertainty between 43 and $45 \mathrm{~mm}$, as opposed to between 45 to $46 \mathrm{~mm}$ as Dwight found. He also found that cartilage added $2 \mathrm{~mm}$ to the diameter, thus indicating a reason for the difference in the indeterminate range, as Dwight used "fresh" specimens. The benefits of Stewart's 1979 humeral study are that it replicates Dwight's results with relative accuracy, with small differences resulting from presence or absence of cartilage, and it is a quick and easy way to determine sex. The issues with this study are that the measurements were taken using different specimen types, fresh and dry, and that the standard developed may not be applicable to other populations, pre-historic populations, or modern populations of the geographic area.

In 2002, Luis Frutos composed a study in order to determine an appropriate metric function standard to estimate sex of a rural Guatemalan forensic sample using the scapula and clavicle. Sixty-two male and 35 female clavicles were used, and 65 male and 38 female scapulas were used in the study. Two clavicular measurements and two scapular measurements were taken. All measurements but the length of the glenoid cavity were used in the computation of a discriminant function on SSPS for Windows using the leave-one-out method due to the small sample size. Frutos' results indicated that male values were greater than female values, with the sex of the individual determined by looking at the sectioning point vs. the discriminant score. If the discriminant score was greater than the sectioning score, then the individual was male; if the discriminant score was less than the sectioning score, then the individual was female. It was found that 85.6 to $94.8 \%$ of individuals were correctly classified by the functions. Frutos then tested the clavicular measurements against North American standards and found very low accuracy in sexing. Therefore, the benefits of Frutos' method are that 
he developed a specific discriminant function for sex determination in a rural Guatemalan population, and others can determine discriminant functions for specific populations based on his method using the clavicle and scapula. The issue with his method is that it is population specific, so if used on other populations the results will be inaccurate.

Frutos comprised another study in 2004 to develop a standard discriminant function for sex estimation of a rural Guatemalan population using the humerus. He used 118 left humeri, from 50 males and 68 females, specimens from rural Guatemalan clandestine graves, which were sexed based on their burial clothes. He measured six humoral dimensions - maximum length, maximum head diameter, mid-shaft circumference, maximum mid-shaft diameter, minimum mid-shaft diameter, and epicondyle breath - using identification and demarcation points from Asala et al, 1998.

From these measurements Frutos computed discriminant functions for each measurement. He found that males were larger than females "He found that males were larger than females in all measurements and that the two best features for sexing were the humeral head diameter with $95.5 \%$ accuracy and the epicondylar breadth with $91.1 \%$ accuracy."

in all measurements and

that the two best features for sexing were the humeral head diameter with $95.5 \%$ accuracy and the epicondylar breadth with $91.1 \%$ accuracy. Sex was determined by comparing the discriminant score with the sectioning point. The discriminant score is the score assigned to each bone, which will then determine which group a bone will belong to when compared to the sectioning score, the cutoff number that determines whether a bone is male or female. If the discriminant score was greater than the sectioning score, then the individual was male; if the discriminant score was less than the sectioning score, then the individual was female. The benefits of Frutos' method are that it is a population specific, quantitative method for sexing a population and other populations can be sexed using a discriminant function computed the same way as Frutos'. The issues with Frutos' method are that it is population specific, thus it cannot be used as a standard for outside populations, and that the determination of sex computed by the discriminant functions were compared to sex determined by the clothes the deceased was wearing, which is not an 
appropriate way to sex an individual in a forensic or archaeological case.

In 2009, Gretchen R. Dabbs reexamined Dwight's scapular length data in order to determine its accuracy. She measured 803 individuals - 308 females and 495 males - from the Hamann-Todd Collection. She found that there was a $96.81 \%$ accuracy in sex determination when the measurement was less than $140 \mathrm{~mm}$ or greater than $170 \mathrm{~mm}$, thus clearly male or female. But, there was only $29.27 \%$ accuracy when the measurement fell between 140 and $170 \mathrm{~mm}$, which is the majority of the modern population. Thus, Dabbs concluded that Dwight's 118-yearold method was not very accurate for current forensic cases due to morphological changes in the population. The benefit of Dabbs' study was that she showed that the current standard used in American forensic cases is inaccurate the majority of the time and that a new standard for modern populations must be developed for sex determination based on the current population.

The use of shoulder girdle components in sex determination of individuals in forensic and archaeological cases is a unique way of sexing when the innomi-

"Only a handful of studies have looked at the shoulder girdle as a way of determining the biological sex of a skeleton." nate and/or skull are not available for sexing. Only a handful of studies have looked at the shoulder girdle as a way of determining the biological sex of a skeleton. Thus, it is an area that has endless possibilities to be explored as long as one keeps in mind that sexing, just like any other means used to identify osteological remains, is population specific. It is clear, based on literature, that the current standards for sexing are inadequate, thus modern standards must be developed in order to accurately identify individuals in modern forensic cases. 


\section{Glossary}

Decedent: An individual who has died.

Discriminant Function: A function of several variates used to assign items into groups.

Endochondrial: The growth or development within cartilage.

Innominate: The two pelvic bones. The pelvis is actually comprised of six bones: two ilium, two ischium, and two pubic bones.

Leave-One-Out Method: A type of statistical cross-validation that uses only a single observation from the original sample in order to validate the data.

Metatarsals: The bones that comprise the main part of one's foot, not including the toes or the heel. There are five in each foot; metatarsal 1 corresponds to the most medial metatarsal, and metatarsal five corresponds to the most lateral metatarsal.

Ontogenesis: The development of an anatomical feature from its earliest developmental stage to maturity.

Ossification: Developmental process of bone formation.

Ossify: To turn into bone or a boney tissue.

Osteological: Pertaining to bone.

Shoulder Girdle: The bones that form one's shoulders; the scapula (shoulder blade), clavicle (collar bone), and proximal (top aspect) of the humerus (upper arm bone).

SSPS for Windows: Statistical Package for the Social Sciences; a computer package for statistical data analysis on a Windows-based computer. 
DOMINACH 
FORBES \& FIFTH 